\title{
Molecular Dynamics simulations of concentrated aqueous electrolyte solutions
}

\author{
Carles Calerd* and Jordi Faraudo \\ Institut de Ciència de Materials de Barcelona (ICMAB-CSIC), Campus de la UAB, E-08193 Bellaterra, Spain \\ Marcel Aguilella-Arzo \\ Biophysics Group, Department of Physics, Universitat Jaume I, 12080 Castelló, Spain.
}

(Dated: May 18, 2010)

\begin{abstract}
Transport properties of concentrated electrolytes have been analyzed using classical molecular dynamics simulations with the algorithms and parameters typical of simulations describing complex electrokinetic phenomena. The electrical conductivity and transport numbers of electrolytes containing monovalent $(\mathrm{KCl})$, divalent $\left(\mathrm{MgCl}_{2}\right)$, a mixture of both $\left(\mathrm{KCl}+\mathrm{MgCl}_{2}\right)$, and trivalent $\left(\mathrm{LaCl}_{3}\right)$ cations have been obtained from simulations of the electrolytes in electric fields of different magnitude. The results obtained for different simulation parameters have been discussed and compared with experimental measurements of our own and from the literature. The electroosmotic flow of water molecules induced by the ionic current in the different cases has been calculated and interpreted with the help of the hydration properties extracted from the simulations.
\end{abstract}

\section{INTRODUCTION}

Understanding the transport properties of electrolytes in aqueous solution is important in a wide range of electrokinetic phenomena such as streaming current experiments 1 , ionic transport in biological and syntetic nanochannels ${ }^{2.3}$ or colloidal electrophoresis. 4.5

Traditionally, electrokinetic and ionic transport phenomena have been described using primitive models in which the solvent is approximated as a continuum of dielectric constant $\epsilon$. Although such models provide an accurate description of a wide variety of phenomena, they fail for cases in which the discrete nature of the solvent plays a fundamental role, or to describe phenomena related to specific hydration of ions.

An alternative theoretical approach to understand electrokinetic phenomena which circumvents the limitations of primitive models is the use of molecular dynamics simulations of ions in explicit solvent. Due to recent improvements in algorithms and computer power, all-atom molecular dynamics simulatios studies of electrokinetic phenomena in some realistic systems are now possible $e^{6,7}$. From such atomistic descriptions, one can elucidate the microscopic mechanisms responsible for macroscopic measurable phenomena. For example, Aksimentiev and Schulten studied at atomic detail the permeation of individual ions through the transmembrane channel $\alpha$-Hemolysin with the help of molecular dynamics simulations ${ }^{6}$. This approach has also been employed to understand from a microscopic perspective the electrophoresis of DNA immersed in multivalent ionic solutions when an external electric field is applied ${ }^{8}$, the transport of monovalent and divalent ions through polymeric and silica nanopores ${ }^{9.10}$, and the ionic selectivity of the $\mathrm{OmpF}$ porin biological nanochannel 11 .

The realistic atomic description of such electrokinetic phenomena involves complex systems containing large number of particles. To be able to cope with such systems using molecular dynamics simulations, the use of algorithms which enhance the computer performance are required. A molecular dynamics simulation package which has been proven to be very successful in the description of biological molecules and elecrokinetic phenomena is NAMD2 $\frac{12}{2}$. To accelerate the calculations of such large systems, in NAMD2 the temperature $\mathrm{T}$ of the system is controlled by using the Langevin thermostat instead of the more rigorous but much more computationally demanding Nosé-Hoover thermostat. Also to speed up the simulations, NAMD2 is usually employed with a multiple time step: a basic timestep for short range interactions and a longer one for the evaluation of $\mathrm{k}$-space contribution to the long range electrostatic forces in the PME technique.

In this paper we study, under different conditions, the transport properties obtained from Molecular Dynamics simulations of electrolyte solutions using the same algorithms and conditions employed in simulations of complex systems. In particular, we focus our analysis on the transport properties of a monovalent electrolyte $(\mathrm{KCl})$, a divalent electrolyte $\left(\mathrm{MgCl}_{2}\right)$, the mixture of both $\left(\mathrm{KCl}+\mathrm{MgCl}_{2}\right)$ and a trivalent electrolyte $\left(\mathrm{LaCl}_{3}\right)$ when an external electric field is applied. While the use of the $\mathrm{KCl}$ electrolyte is standard in molecular dynamics studies and its transport properties have been briefly analyzed from this perspective before ${ }^{\underline{6}}$, the reliability of such simulations methods in dealing with transport properties of multivalent electrolytes have not been tested in detail in spite of the rich phenomenology that they originate $\frac{1,2.13,14}{}$ and their use in the simulation of electrokinetic phenomena involving divalent and trivalent ions ${ }^{8,15}-17$.

\section{METHODS}

\section{A. Simulation Methods}

We have studied the transport properties of different electrolytes by performing molecular dynamics simulations under external electric fields of different magnitudes. The systems considered were ionic solutions of $\mathrm{KCl}, \mathrm{MgCl}_{2}$, a mixture of $\mathrm{KCl}$ and $\mathrm{MgCl}_{2}$, and $\mathrm{LaCl}_{3}$, see Table I. To study the effect of the system size on the ionic transport properties of bulk electrolytes extracted from molecular dynamics simulations, two cubic simulation boxes of different size were used, $L \simeq 4 \mathrm{~nm}$ and $L \simeq 8 \mathrm{~nm}$. 
All simulations have been carried out with the molecular dynamics simulation package NAMD $2 \frac{12}{}$, since it is widely employed in the simulation of biological macromolecules. Water was described using the TIP3P water model as implemented by the CHARMM force field. The ions were modeled as charged Lennard-Jones particles with parameters given by the CHARMM force field for $\mathrm{K}^{+}, \mathrm{Mg}^{2+}$ and for $\mathrm{Cl}^{-}$, while the Lennard-Jones parameters for $\mathrm{La}^{3+}$ were taken from Ref 18 .

The initial configuration of the simulation was constructed as follows. The ions $\left(\mathrm{K}^{+}, \mathrm{Mg}^{2+}\right.$ and $\left.\mathrm{Cl}^{-}\right)$were inserted at random positions by employing the AutoIonize plugin of the Visual Molecular Dynamics (VMD) software package $\frac{19}{9}$ inside a cube of preequilibrated TIP3P water molecules, obtained with the help of the Solvate plugin of VMD (see Fig. 1 for a diagram of the system). The faces of the cube are parallel to the $\mathrm{XY}, \mathrm{XZ}$, and $\mathrm{YZ}$ planes. In all simulations we employed periodic boundary conditions in all directions. Lennard-Jones interactions were computed using a smooth (10-12 Å) cutoff, as it is customary done in NAMD2 simulations $\frac{6.8}{}$. The electrostatic interactions were calculated using the particlemesh Ewald (PME) method with a precision of $10^{-6}$ using a $128 \times 128 \times 128$ grid and a 12 Åcutoff for the real space calculation. These are common parameters used to simulate complex and large biological macromolecules ${ }^{6}$.

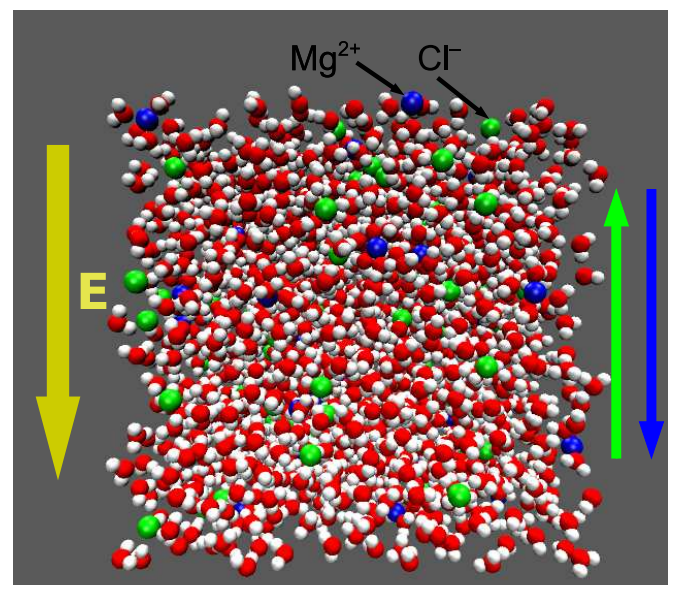

FIG. 1: Snapshot of a simulation of the $1 \mathrm{M} \mathrm{MgCl}$ electrolyte with simulation box size of $L=3.82 \mathrm{~nm}$. Chloride ions are represented in green, magnesium ions in blue, and water molecules in red (oxygen) and white (hydrogen). The yellow arrow points in the direction of the applied electric field, the green arrow in the direction of the flow of chloride ions (anions), and the blue arrow in the direction of the flow of magnesium ions (cations).

For each case, the equilibration procedure consisted of 50000 steps of energy minimization, a $1 \mathrm{~ns}$ run in the $\mathrm{NpT}$ ensemble (with $p=1 \mathrm{~atm}$ and $T=296 \mathrm{~K}$ ) followed by a $1 \mathrm{~ns}$ run in the NVT ensemble $(T=296 \mathrm{~K})$. Production runs in the NVT ensemble were performed in the presence of different electric fields to induce electromigration of the ions in solution. The NpT simulation runs were performed employing a combination of the Nosé-Hoover constant pressure method with the piston fluctuation control implemented using Langevin dynamics as implemented in NAMD2 $(p=1 \mathrm{~atm}$, period of the oscillations of $0.1 \mathrm{ps}$ and relaxation constant of $0.05 \mathrm{ps}$ ). As mentioned above, to speed up calculations the NVT runs were carried out by applying Langevin dynamics, with parameters (also in the NpT run) $T=296 \mathrm{~K}$ and a damping coefficient of $1 \mathrm{ps}^{-1}$ (using $0.2 \mathrm{ps}^{-1}$ to test its effect in the dynamics for some cases specified later). Langevin forces were applied to all atoms except for hydrogens, which thermalize through interactions with the rest of the system.

In all cases, the equations of motion were solved using a multiple time step in order to speed up the simulations. A basic time step of $2 \mathrm{fs}$ was used for the evaluation of short range interactions and a longer time step of $4 \mathrm{fs}$ for the calculations of the $\mathrm{k}$-space contribution to the long range electrostatic forces in the PME technique.

In the production runs, the instantaneous current induced by the external electric field applied along the Z-direction is calculated with the help of t $^{\underline{6}}$

$$
I(t)=\frac{1}{\Delta t L} \sum_{i=1}^{N} q_{i}\left[z_{i}(t+\Delta t)-z_{i}(t)\right],
$$

where $z_{i}$ and $q_{i}$ are the Z-coordinate and the charge of atom $i$, respectively. $L$ is the size of the simulation box and $\Delta t$ is the time interval employed to record data, which was chosen to be $\Delta t=10 \mathrm{ps}$. The average current is computed by linearly fitting the cumulative current that is obtained by integration of the instantaneous current. To ensure constistency of the results, the current was also computed by counting the flux of ions crossing a plane perpendicular to the direction of the electric field and located in the center of the simulation box. The conductivity $\kappa$ of the solution is defined by:

$$
\frac{I}{A}=\kappa E
$$

where $A=L^{2}$ is the cross section area perpendicular to the electric field $E$. In order to obtain the conductivity of the electrolyte, we have performed simulations at different electric fields $E$ and calculated the current $I$ induced by them. In all cases, an ohmic behaviour has been observed (i.e. consistent with a linear relation between $I$ and $E$ ), and a linear fit of the data to Eq. (2) gives the value of the conductivity $\kappa$.

The electromigration of ions in aqueous solutions as a result of the application of an external electric field is often accompanied by a net flow of water, the so-called electroosmotic flow. The electroosmotic flow has been evaluated by keeping track, every $\Delta t=10 \mathrm{ps}$, of the accumulated number of water molecules crossing a plane perpendicular to the electric field. Crossings of such plane in the direction of the electric field are counted as positive, whereas crossings in the opposite direction are counted as negative. Hence, the sign of the overall flow gives the direction of the electroosmotic flow with respect to the elecric field direction. As reported before ${ }^{6}$, the simulations might give a drift of the whole system which is unphysical since no net force is applied to the system (the electrolyte is globally electrically neutral). To avoid such spurious effects, the computation of the electroosmotic flow has 
TABLE I: Simulation parameters for the simulations performed of the different electrolytes: cubic box edge length (L), damping constant of the Langevin thermostat $\left(\tau_{\mathrm{Lan}}\right)$, number of $\mathrm{K}^{+}$ions, number of $\mathrm{Mg}^{2+}$ ions, number of $\mathrm{La}^{3+}$ ions, number of $\mathrm{Cl}^{-}$ions, number of water molecules, simulation time, external electric field applied, and potential difference between the edges of the cubic box along the direction of the electric field.

\begin{tabular}{|c|c|c|c|c|c|c|c|c|c|c|}
\hline Electrolyte & $\mathrm{L}(\mathrm{nm})$ & $\tau_{\text {Lan }}\left(\mathrm{ps}^{-1}\right)$ & $\begin{array}{l}\text { Num. } \\
\mathrm{K}^{+}\end{array}$ & $\begin{array}{l}\text { Num. } \\
\mathrm{Mg}^{2+}\end{array}$ & $\begin{array}{l}\text { Num. } \\
\mathrm{La}^{3+}\end{array}$ & $\begin{array}{l}\mathrm{Num} . \\
\mathrm{Cl}^{-}\end{array}$ & $\begin{array}{l}\mathrm{Num} . \\
\mathrm{H}_{2} \mathrm{O}\end{array}$ & $\begin{array}{l}\text { Simulation } \\
\text { time (ns) }\end{array}$ & $\begin{array}{l}\text { Electric } \\
\text { field }(\mathrm{mV} / \mathrm{nm})\end{array}$ & $\Delta \mathrm{V}(\mathrm{mV})$ \\
\hline \multirow[t]{8}{*}{$1 \mathrm{M} \mathrm{KCl}$} & 3.88 & 1.0 & 37 & 0 & 0 & 37 & 1907 & 21.0 & 14.2 & 55 \\
\hline & 3.88 & 1.0 & 37 & 0 & 0 & 37 & 1907 & 9.0 & 26.0 & 109 \\
\hline & 3.88 & 1.0 & 37 & 0 & 0 & 37 & 1907 & 9.0 & 43.3 & 168 \\
\hline & 3.88 & 1.0 & 37 & 0 & 0 & 37 & 1907 & 9.0 & 86.6 & 336 \\
\hline & 7.82 & 1.0 & 306 & 0 & 0 & 306 & 15774 & 9.0 & 14.2 & 111 \\
\hline & 7.82 & 1.0 & 306 & 0 & 0 & 306 & 15774 & 9.0 & 26.0 & 203 \\
\hline & 7.82 & 1.0 & 306 & 0 & 0 & 306 & 15774 & 9.0 & 43.3 & 339 \\
\hline & 7.82 & 1.0 & 306 & 0 & 0 & 306 & 15774 & 9.0 & 86.6 & 677 \\
\hline \multirow[t]{12}{*}{$1 \mathrm{M} \mathrm{MgCl}_{2}$} & 3.82 & 1.0 & 0 & 37 & 0 & 74 & 1870 & 9.0 & 14.2 & 54 \\
\hline & 3.82 & 1.0 & 0 & 37 & 0 & 74 & 1870 & 9.0 & 26.0 & 99 \\
\hline & 3.82 & 1.0 & 0 & 37 & 0 & 74 & 1870 & 9.0 & 43.3 & 165 \\
\hline & 3.82 & 1.0 & 0 & 37 & 0 & 74 & 1870 & 9.0 & 86.6 & 331 \\
\hline & 7.73 & 1.0 & 0 & 306 & 0 & 612 & 15468 & 9.0 & 14.2 & 110 \\
\hline & 7.73 & 1.0 & 0 & 306 & 0 & 612 & 15468 & 9.0 & 26.0 & 201 \\
\hline & 7.73 & 1.0 & 0 & 306 & 0 & 612 & 15468 & 9.0 & 43.3 & 335 \\
\hline & 7.73 & 1.0 & 0 & 306 & 0 & 612 & 15468 & 9.0 & 86.6 & 669 \\
\hline & 7.73 & 0.2 & 0 & 306 & 0 & 612 & 15468 & 9.0 & 14.2 & 110 \\
\hline & 7.73 & 0.2 & 0 & 306 & 0 & 612 & 15468 & 9.0 & 26.0 & 201 \\
\hline & 7.73 & 0.2 & 0 & 306 & 0 & 612 & 15468 & 9.0 & 43.3 & 335 \\
\hline & 7.73 & 0.2 & 0 & 306 & 0 & 612 & 15468 & 9.0 & 86.6 & 669 \\
\hline $0.11 \mathrm{M} \mathrm{MgCl}_{2}$ & 7.83 & 1.0 & 0 & 31 & 0 & 62 & 16293 & 9.0 & 43.3 & 339 \\
\hline $0.33 \mathrm{M} \mathrm{MgCl}_{2}$ & 7.80 & 1.0 & 0 & 93 & 0 & 186 & 16107 & 9.0 & 43.3 & 338 \\
\hline $0.54 \mathrm{M} \mathrm{MgCl}_{2}$ & 7.79 & 1.0 & 0 & 155 & 0 & 310 & 15921 & 9.0 & 43.3 & 337 \\
\hline $1 \mathrm{M} \mathrm{MgCl}{ }_{2}$ & 7.72 & 1.0 & 306 & 306 & 0 & 918 & 14856 & 9.0 & 14.2 & 110 \\
\hline \multirow[t]{3}{*}{ + 1MKCl } & 7.72 & 1.0 & 306 & 306 & 0 & 918 & 14856 & 9.0 & 26.0 & 201 \\
\hline & 7.72 & 1.0 & 306 & 306 & 0 & 918 & 14856 & 9.0 & 43.3 & 334 \\
\hline & 7.72 & 1.0 & 306 & 306 & 0 & 918 & 14856 & 9.0 & 86.6 & 668 \\
\hline \multirow[t]{4}{*}{$1 \mathrm{M} \mathrm{LaCl}_{3}$} & 7.71 & 1.0 & 0 & 0 & 308 & 924 & 15154 & \begin{tabular}{|l}
13.19 \\
\end{tabular} & 14.2 & 110 \\
\hline & 7.71 & 1.0 & 0 & 0 & 308 & 924 & 15154 & 10.87 & 26.0 & 201 \\
\hline & 7.71 & 1.0 & 0 & 0 & 308 & 924 & 15154 & 10.12 & 43.3 & 334 \\
\hline & 7.71 & 1.0 & 0 & 0 & 308 & 924 & 15154 & 10.52 & 86.6 & 668 \\
\hline
\end{tabular}

been done in the frame of reference of the center of mass of the whole system.

The electric current flowing in an electrolyte solution is caused by the motion of anions and cations moving in opposite directions under the applied field. The fraction of the total current induced by each ion type defines its transport number, which is in general a function of the electrolyte concentration. The fraction of electrical current carried by cations defines the cationic transport number, $\mathrm{t}_{+}$, and the fraction of the electrical current carried by anions the anionic transport number, $\mathrm{t}_{-}$. A completely equivalent quantity to transport numbers is the ratio of the current induced by anions (anionic current) over the current induced by cations (cationic current), which will be be refered as the ratio of currents throughout the paper. Due to the global electroneutrality of the system, the total electric current flowing through an electrolyte as a result of the application of an external electric field is independent of the frame of reference in which it is measured. The ratio of currents and transport numbers, however, are frame dependent, and their computation must be done carefully. A proper account of the contribution to the total current from every ion in electrolyte solutions is important to describe phenomena in other more complex systems in which not only the total electric current but also the flow of each type of ion is relevant (e.g. in the study of the selectivity of ionic channels). Experimentally, the relevant frame of reference in which data of transport numbers and ratio of currents is provided is the frame of reference of the moving fluid. Therefore, to facilitate the comparison with experimental values, transport numbers and ratio of currents will be given in the frame of reference comoving with 
the fluid.

\section{B. Conductivity measurements}

The electrolyte conductivity measurements were performed using a MeterLab CDM210 (R) conductivity meter, Radiometer Analytical SAS (France). The solutions were prepared using water from a Water Purification System Millipore Simplicity 185. Magnesium Chloride 6-hydrated $(\mathrm{MgCl} 2)$ and Potassium Chloride $(\mathrm{KCl})$ from Panreac in all cases following ACS specifications. Weighting of the compounds were done with a Mettler Toledo AB104-S, in the quantities necessary to achieve a $1 \mathrm{M}$ concentration. The conductivity measurement included a stirring of the solution with a magnetic stirrer and heater JPSelecta Agimatic-E, with temperature monitoring using a laboratory thermometer at $296.0 \mathrm{~K}$.

\section{RESULTS}

\section{A. A test case: Transport properties of $1 \mathrm{M} \mathrm{KCl}$}

The results obtained from molecular dynamics simulations for the electrical properties of the $1 \mathrm{M} \mathrm{KCl}$ electrolyte are given in Fig. 2 and Table We have used two different sizes for the simulation box, $L=3.88 \mathrm{~nm}$ and $L=7.82 \mathrm{~nm}$, to test the dependence of the results on the simulation's box size. As can be seen, the values for the ionic conductivity for the two different box sizes do not differ significantly, $\kappa=13.4 \mathrm{~S} / \mathrm{m}$ for the small box and $\kappa=12.6 \mathrm{~S} / \mathrm{m}$ for the big one. Both results are in good agreement with our measured experimental value $\kappa_{\exp }=11.24 \pm 0.01 \mathrm{~S} / \mathrm{m}$, being the value of the larger system closer to the experimental result as it should be expected. Note that in previous studies of $1 \mathrm{M} \mathrm{KCl}$ bulk electrolyte (see Ref. ${ }^{6}$ ), it was argued that a $0.2 \mathrm{ps}^{-1}$ damping constant is necessary in order to reproduce correctly transport properties. However, our present results were obtained using a Langevin thermostat with a damping constant of $1 \mathrm{ps}^{-1}$, which is the typical choice for simulations of complex systems in contact with electrolyte (such as protein channels or silica nanochannels ${ }^{6,10,11}$ ). Our results show that with the standard simulation parameters employed in complex systems the conductivity of the electrolyte $\mathrm{KCl}$ is correctly reproduced.

We have also calculated the ratio of the different contributions to the total current from anions and cations (equivalent to the ratio of transport numbers). As explained in the previous section, it is defined as the ratio of the anionic and cationic currents in the frame of reference of the moving fluid. The results for such ratio of currents are given in Table W As shown in the table, the values calculated from molecular dynamics simulations are in good agreement with the experimental value of $I_{\mathrm{Cl}} / I_{\mathrm{K}}=1.048$ (equivalent to a value of the cation transport number $t_{+}=0.488^{20}$ ) for both sizes of the cubic simulation box. (a)

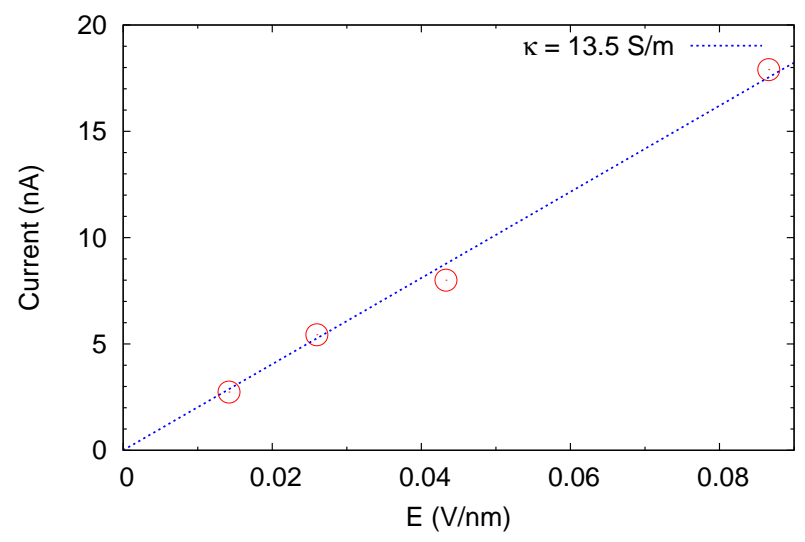

(b)

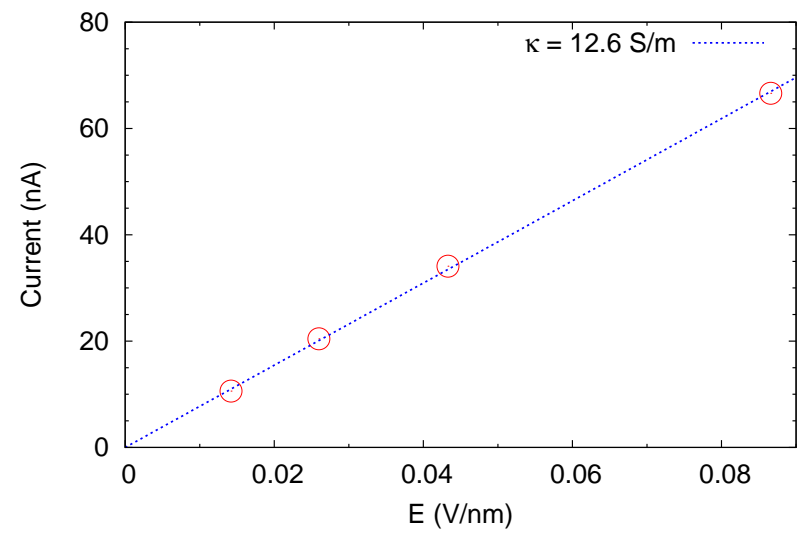

FIG. 2: Electric current versus the applied electric field for a $1 \mathrm{M} \mathrm{KCl}$ electrolyte in simulations using two different simulation box sizes $L$. The points correspond to simulation data computed from Eq. (1) and lines are fits of the data to Eq.(2) (a) $L=3.88 \mathrm{~nm}$ (fit gives $\kappa=13.4 \mathrm{~S} / \mathrm{m}$ ).(b) $L=7.82 \mathrm{~nm}$ (fit gives $\kappa=12.6 \mathrm{~S} / \mathrm{m}$ ).

\section{Electoosmotic flow}

In addition to ionic currents, we also observe a net flow of water molecules. In Fig. 3, the accumulated number of water molecules per unit area crossing a plane perpendicular to the electric field is represented as a function of simulation time. A linear fit of these magnitudes provides the flux of water molecules for every value of the electric field, as shown in Table [II As shown in Fig. 3 and Table III the electroosmotic flow for the $1 \mathrm{M} \mathrm{KCl}$ electrolyte is in the direction opposite to the external field, that is, in the direction of the flow of chloride ions.

\section{B. Transport properties of $1 \mathrm{M} \mathrm{MgCl}_{2}$}

A similar procedure has been followed to obtain the transport properties of a $1 \mathrm{M} \mathrm{MgCl}_{2}$ electrolyte. The electric current induced by the electromigration of ions was obtained for different values of the electric field using two different sizes of 
TABLE II: Ionic contributions to the total current, ratio of the anionic current over the cationic current and cationic transport numbers for different values of the applied electric field in the case of a $1 \mathrm{M} \mathrm{KCl}$ electrolyte. All these quantities are calculated in the frame of reference of the moving fluid.

\begin{tabular}{|c|c|c|c|c|c|}
\hline \hline System Size & Electric field $(\mathrm{mV} / \mathrm{nm})$ & $\mathrm{I}_{\mathrm{Cl}}(\mathrm{nA})$ & $\mathrm{I}_{\mathrm{K}}(\mathrm{nA})$ & $\mathrm{I}_{\mathrm{Cl}} / \mathrm{I}_{\mathrm{K}}$ & $\mathrm{t}_{+}$ \\
\hline \multirow{5}{*}{$\mathrm{L}=3.88 \mathrm{~nm}$} & 14.2 & 1.43 & 1.39 & 1.03 & 0.49 \\
& 25.98 & 2.80 & 2.59 & 1.08 & 0.48 \\
& 43.3 & 4.28 & 3.83 & 1.12 & 0.47 \\
& 86.6 & 8.42 & 8.22 & 1.02 & 0.49 \\
\hline \multirow{5}{*}{$\mathrm{L}=7.82 \mathrm{~nm}$} & 14.2 & 5.49 & 5.14 & 1.07 & 0.48 \\
& 25.98 & 10.35 & 10.11 & 1.02 & 0.49 \\
& 43.3 & 17.02 & 17.16 & 1.00 & 0.50 \\
& 86.6 & 33.57 & 33.17 & 1.01 & 0.49 \\
\hline
\end{tabular}

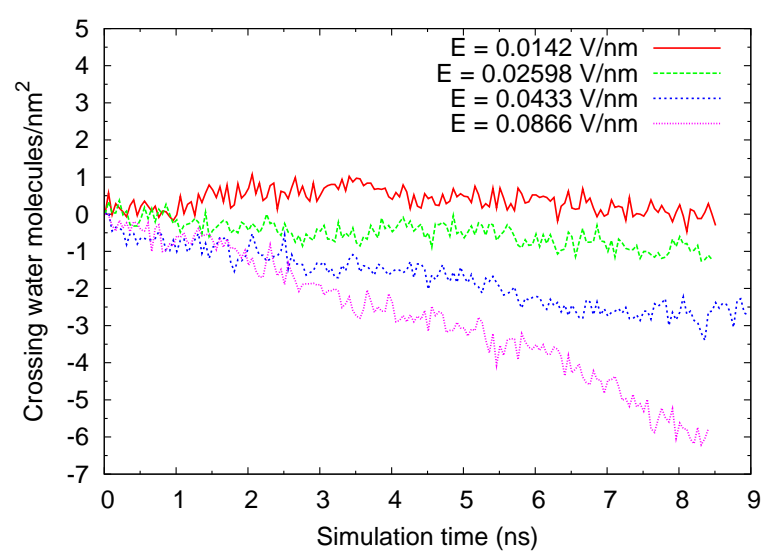

FIG. 3: Accumulated number of water molecules per unit area crossing a plane perpendicular to the applied electric field versus simulation time for the $1 \mathrm{M} \mathrm{KCl}$ electrolyte. Different lines represent the flow of water for different values of the electric field.

a cubic simulation box, $L=3.82 \mathrm{~nm}$ and $L=7.73 \mathrm{~nm}$. The results of the simulations are given in Figs. 4 and Table IV. The values for the ionic conductivity of the two different box sizes do not differ significantly, being $\kappa=14.2 \mathrm{~S} / \mathrm{m}$ for the small box and $\kappa=11.9 \mathrm{~S} / \mathrm{m}$ for the big one. Both results agree well with experimental values, being the result from the larger simulation box much more accurate. According to Phang and Stokes ${ }^{21}, \kappa_{\exp }=11.4 \mathrm{~S} / \mathrm{m}$ at $\left[\mathrm{MgCl}_{2}\right]=0.9674$ $\mathrm{M}$ and $T=298.15 \mathrm{~K}$. A recent critical review 22 provides a sligthly larger value of $\kappa_{\exp }=11.6 \mathrm{~S} / \mathrm{m}$ at $\left[\mathrm{MgCl}_{2}\right]=1$ $\mathrm{M}$ and $T=25^{\circ} \mathrm{C}$. Our own measurements give a value of $\kappa_{\text {exp }}=11.92 \mathrm{~S} / \mathrm{m}$.

The ratio between the different contributions of anions and cations to the total current has been evaluated. The results for such ratio of currents are given in Table IV] These results emphasize the need for using a large enough simulation box. The values for the ratio of currents and transport numbers obtained for the cubic simulation box of size $L=3.82 \mathrm{~nm}$ exhibit a spurious dependence on the applied electric field. For the larger simulation box, the results are not field dependent, as should
TABLE III: Flux of water molecules for the different electrolytes and different values of the external electric field.

\begin{tabular}{|c|c|c|}
\hline Electrolyte & Electric field $(\mathrm{mV} / \mathrm{nm})$ & Water flux $\left(\mathrm{nm}^{-2} \mathrm{~ns}^{-1}\right)$ \\
\hline $1 \mathrm{M} \mathrm{KCl}$ & 14.2 & $0.12 \pm 0.13$ \\
& 26.0 & $-0.13 \pm 0.04$ \\
& 43.3 & $-0.43 \pm 0.14$ \\
& 86.6 & $-0.64 \pm 0.07$ \\
\hline $1 \mathrm{M} \mathrm{MgCl}{ }_{2}$ & 14.2 & $0.76 \pm 0.04$ \\
& 26.0 & $1.47 \pm 0.05$ \\
& 43.3 & $2.30 \pm 0.14$ \\
& 86.6 & $5.11 \pm 0.09$ \\
\hline $1 \mathrm{M} \mathrm{KCl}+1 \mathrm{M} \mathrm{MgCl}_{2}$ & 14.2 & $0.43 \pm 0.15$ \\
& 26.0 & $0.88 \pm 0.06$ \\
& 43.3 & $1.31 \pm 0.07$ \\
& 86.6 & $2.82 \pm 0.23$ \\
\hline $1 \mathrm{M} \mathrm{LaCl}_{3}$ & 14.2 & $-0.25 \pm 0.11$ \\
& 26.0 & $-0.28 \pm 0.25$ \\
& 43.3 & $-0.33 \pm 0.08$ \\
& 86.6 & $-0.89 \pm 0.24$ \\
\hline
\end{tabular}

TABLE IV: Ionic contributions to the total current, ratio of the anionic current over the cationic current and cationic transport numbers for different values of the applied electric field in the case of a $1 \mathrm{M}$ $\mathrm{MgCl}_{2}$ electrolyte. All these quantities are calculated in the frame of reference of the moving fluid.

\begin{tabular}{|c|c|c|c|c|c|}
\hline \hline $\begin{array}{c}\text { System } \\
\text { Size }\end{array}$ & $\begin{array}{c}\text { Electric } \\
\text { field }(\mathrm{mV} / \mathrm{nm})\end{array}$ & $\mathrm{I}_{\mathrm{Cl}}(\mathrm{nA})$ & $\mathrm{I}_{\mathrm{Mg}}(\mathrm{nA})$ & $\mathrm{I}_{\mathrm{Cl}} / \mathrm{I}_{\mathrm{Mg}}$ & $\mathrm{t}_{+}$ \\
\hline & 14.2 & 1.60 & 0.72 & 2.23 & 0.31 \\
$\mathrm{~L}=3.82 \mathrm{~nm}$ & 25.98 & 3.09 & 2.22 & 1.39 & 0.42 \\
& 43.3 & 5.63 & 3.56 & 1.58 & 0.39 \\
& 86.6 & 10.52 & 7.42 & 1.42 & 0.41 \\
\hline & 14.2 & 5.69 & 4.13 & 1.38 & 0.42 \\
$\mathrm{~L}=7.73 \mathrm{~nm}$ & 25.98 & 11.31 & 8.24 & 1.37 & 0.42 \\
& 43.3 & 18.05 & 13.66 & 1.32 & 0.43 \\
& 86.6 & 35.06 & 26.30 & 1.33 & 0.43 \\
\hline
\end{tabular}

be expected. Such results show that the anionic contribution to the current is significantly larger than the cationic contribution (being a factor of 1.4 between them). In our simulations these differences in the anionic and cationic contributions to the current can be attributed, to a large extent, to differences in diffusion coefficients between both ions. In order to disentangle the diffusional and correlation contributions to the transport number, we have evaluated the translational diffusion coefficient of each ion by computing the mean square displacement of each ion in a 2 ns long NVE simulation run with no external field applied. The results of $D_{\mathrm{Mg}}=0.95 \times 10^{-5} \mathrm{~cm}^{2} / \mathrm{s}$ and $D_{\mathrm{Cl}}=1.69 \times 10^{-5} \mathrm{~cm}^{2} / \mathrm{s}$ for the diffusion coefficients of $\mathrm{Mg}^{2+}$ and $\mathrm{Cl}^{-}$, respectively, lead to a ratio between the diffusion coefficients of $D_{\mathrm{Cl}} / D_{\mathrm{Mg}}=1.8$.

Experimentally, both diffusion coefficients and transport numbers can be obtained, so we can compare both simulation results with experimental data. Using NMR, Struis et al $\stackrel{23}{\underline{23}} \mathrm{ob}-$ 
(a)

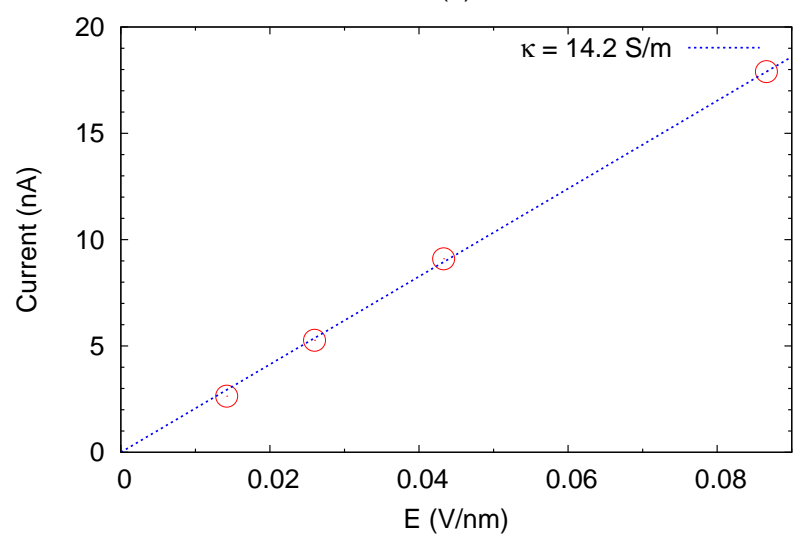

(b)

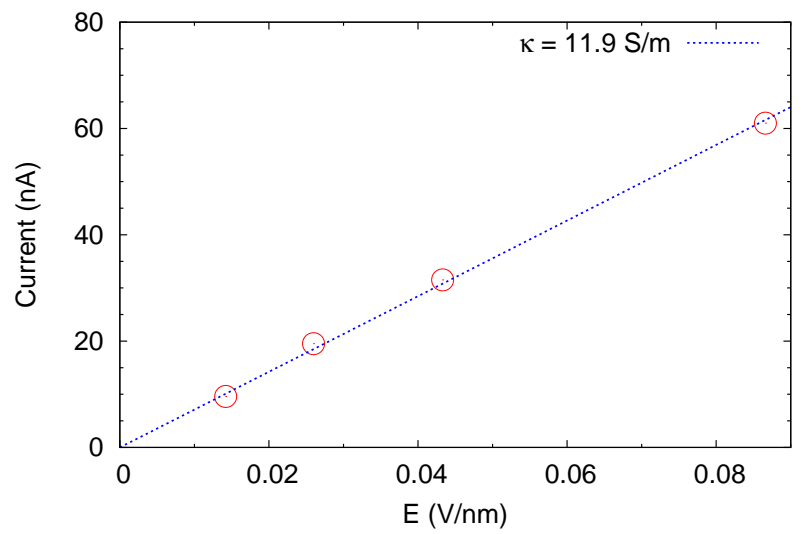

FIG. 4: Electric current versus applied electric field for a $1 \mathrm{M} \mathrm{MgCl}_{2}$ electrolyte in simulations using two different simulation box sizes $L$. The points correspond to simulation data computed from Eq.(1) and lines are fits of the data to Eq.(2) (a) $L=3.82 \mathrm{~nm}$ size cubic simulation box (fit gives $\kappa=14.2 \mathrm{~S} / \mathrm{m}$ ). (b) $L=7.73 \mathrm{~nm}$ (fit gives $\kappa=11.9 \mathrm{~S} / \mathrm{m})$

tained $D_{\mathrm{Mg}}=0.93 \times 10^{-5} \mathrm{~cm}^{2} / \mathrm{s}$ and $D_{\mathrm{Cl}}=2.94 \times 10^{-5}$ $\mathrm{cm}^{2} / \mathrm{s}$ (at $25^{\circ} \mathrm{C}$ and $0.985 \mathrm{~mol} / \mathrm{Kg}$ concentration), so the experimental ratio is $D_{\mathrm{Cl}} / D_{\mathrm{Mg}}=3.16$. The simulations reported here reproduce with good accuracy the diffusion coefficient for $\mathrm{Mg}^{2+}$ but the diffusion coefficient for $\mathrm{Cl}^{-}$is greatly underestimated. Concerning transport numbers, electrochemical measurements ${ }^{21,22}$ give a transport number for cations around 0.3 , so the experimental ratio between anionic and cationic currents is $\simeq 2.3$. The difference between the ratio of transport numbers obtained by electrochemical methods and the ratio between diffusion coefficients obtained by NMR can be interpreted in several ways. First of all, thermodynamic arguments ${ }^{24}$ show that some experimental procedures mixed up different reference frames, so the electrochemical results have to be interpreted with caution. If the difference between both ratios is indeed physical, the difference can be attributed to electrokinetic processes (not accounted by diffusion coefficients) which are supposed to affect the transport numbers of each ion ${ }^{20}$. In any case, these electrokinetic processes were predicted in the framework of classical, continuum theory of

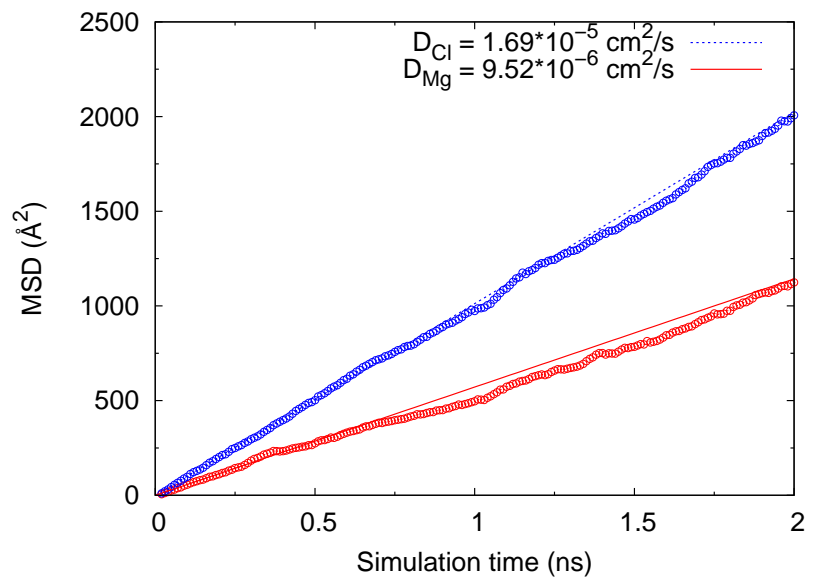

FIG. 5: Diffusion of $\mathrm{Mg}^{2+}$ and $\mathrm{Cl}^{-}$ions in $1 \mathrm{M}$ aqueous solution as obtained from 2ns NVE simulations with a cubic simulation box of $L=7.73 \mathrm{~nm}$.

TABLE V: Ionic contributions to the total current, ratio of the anionic current over the cationic current and cationic transport numbers for different values of the applied electric field in the case of a $1 \mathrm{M}$ $\mathrm{MgCl}_{2}$ electrolyte, $L=7.73 \mathrm{~nm}$, and using a damping constant of the Langevin thermostat of $0.2 \mathrm{ps}^{-1}$. All these quantities are calculated in the frame of reference of the moving fluid.

\begin{tabular}{|c|c|c|c|c|}
\hline \hline Electric field $(\mathrm{V} / \mathrm{nm})$ & $\mathrm{I}_{\mathrm{Cl}}(\mathrm{nA})$ & $\mathrm{I}_{\mathrm{Mg}}(\mathrm{nA})$ & $\mathrm{I}_{\mathrm{Cl}} / \mathrm{I}_{\mathrm{Mg}}$ & $\mathrm{t}_{+}$ \\
\hline 14.2 & 5.88 & 5.25 & 1.12 & 0.47 \\
25.98 & 11.49 & 9.56 & 1.20 & 0.45 \\
43.3 & 19.82 & 15.03 & 1.32 & 0.43 \\
86.6 & 38.59 & 31.15 & 1.24 & 0.45 \\
\hline
\end{tabular}

electrolytes and are not clearly observed in our simulations, so its molecular origin remains unclear.

The effect of the damping constant of the Langevin thermostat was investigated by performing molecular dynamics simulations on the same system of size $L=7.73 \mathrm{~nm}$ and same conditions but using a Langevin thermostat with a damping constant of $\tau_{\text {Lan }}=0.2 \mathrm{ps}^{-1}$ (instead of the damping constant of $1 \mathrm{ps}^{-1}$ employed in all other simulations). The electrolyte exhibits an ohmic behaviour, with conductivity $\kappa=13.5 \mathrm{~S} / \mathrm{m}$, which slightly differs from the value obtained before and from the experimental value. Nevertheless, the major effect of the damping constant is in the different ionic contributions to the total current, as can be seen in Fig. V. In comparison to the results of the simulations with $\tau_{\text {Lan }}=\mathrm{ps}^{-1}$, it is evident that there is a spurious dependence of the ratio of the anionic current over the cationic current on the magnitude of the applied electric field.

\section{Electoosmotic flow}

The electroosmotic flow induced by the ionic current was also computed. In Fig. 6, the accumulated number of water molecules per unit area crossing a plane perpendicular to the 
TABLE VI: Hydration of the different ions

\begin{tabular}{|c|c|c|c|c|c|}
\cline { 4 - 6 } & \multicolumn{3}{c|}{ Number of attached $\mathrm{H}_{2} \mathrm{O}$} \\
\hline Electrolyte & Ion & Hydration & $\tau=10 \mathrm{ps}$ & $\tau=100 \mathrm{ps}$ & $\tau=1000 \mathrm{ps}$ \\
\hline \multirow{2}{*}{$1 \mathrm{M} \mathrm{KCl}$} & $\mathrm{K}^{+}$ & 6.4 & 1.88 & 0.070 & 0.0012 \\
& $\mathrm{Cl}^{-}$ & 7.3 & 2.41 & 0.11 & 0.0033 \\
\hline \multirow{2}{*}{$1 \mathrm{M} \mathrm{MgCl}_{2}$} & $\mathrm{Mg}^{2+}$ & 6.0 & 5.41 & 5.35 & 5.31 \\
& $\mathrm{Cl}^{-}$ & 7.3 & 2.99 & 0.19 & 0.005 \\
\hline \multirow{3}{*}{$1 \mathrm{M} \mathrm{KCl} \& 1 \mathrm{M} \mathrm{MgCl}_{2}$} & $\mathrm{Mg}^{2+}$ & 6.0 & 5.33 & 5.22 & 5.13 \\
& $\mathrm{~K}^{+}$ & 6.9 & 1.95 & 0.11 & 0.003 \\
& $\mathrm{Cl}^{-}$ & 7.0 & 3.04 & 0.25 & 0.009 \\
\hline \multirow{2}{*}{$1 \mathrm{M} \mathrm{LaCl}$} & $\mathrm{La}^{3+}$ & 8.4 & 6.98 & 4.65 & 0.27 \\
& $\mathrm{Cl}^{-}$ & 6.9 & 3.50 & 0.35 & 0.009 \\
\hline
\end{tabular}

electric field is represented as a function of simulation time. The flux of water molecules for each electric field is given in Table IIII It is interesting to note the different direction and magnitude of the water flow obtained for $1 \mathrm{M} \mathrm{KCl}$ and for $1 \mathrm{M}$ $\mathrm{MgCl}_{2}$ electrolytes. Indeed, while in the presence of $1 \mathrm{M} \mathrm{KCl}$ the direction of the net flow of water is opposite to the direction of the electric field, in the presence of $1 \mathrm{M} \mathrm{MgCl}_{2}$ the net flow of water goes in the direction of the applied field. The magnitude of the net flow of water also differs significantly in both cases, being much larger (almost an order of magnitude) for the $1 \mathrm{M} \mathrm{MgCl}_{2}$ electrolyte. These differences can be understood from the hydration properties of the ions involved. For each ion, we have computed its hydration, i.e., the average number of water molecules in its first coordination shell (as defined by the first minimum of the radial distribution function, see Figs. 7 and 8 . We have also computed the average number of water molecules which have remained a time $\tau$ in the first coordination shell of each ion to test the robustness of the hydration layer, see Table VI The values given in Table VI do not show any dependence on the value of the electric field applied in the simulations. The reported results for the hydration values of the different ions agree with results from previous molecular dynamics studies $5-27$ and $\mathrm{Ab}$ initio calculations ${ }^{28}$. The results for the average number of water molecules that spend a time $\tau$ in the first coordination shell of the different ions are also in agreement with previous computational studies, in which residence times of water molecules in the first coordination shell of the order of $\sim 10 \mathrm{ps}$ were obtained for $\mathrm{K}^{+}$and $\mathrm{Cl}^{-}$and of the order of $\sim 10 \mathrm{~ns}$ for $\mathrm{Mg}^{2+}$. Such difference in the robustness of the hydration layer of $\mathrm{Mg}^{2+}$ and the other ions is a broadly accepted fact, established by ab initio and DFT calculations s $28,29^{2}$ as well as by NMR, Raman spectroscopy, and X-ray adsorption spectroscopy experiments ${ }^{23,30,31}$.

Hence, from the analysis of the hydration properties of the ions it is possible to understand the difference in the electroosmotic flow between the $1 \mathrm{M} \mathrm{KCl}$ and $1 \mathrm{M} \mathrm{MgCl}_{2}$ electrolytes. For the $1 \mathrm{M} \mathrm{KCl}$ electrolyte, the anionic current is slightly greater than the cationic current and the hydration of $\mathrm{Cl}^{-}$is higher than that of $\mathrm{K}^{+}$so it exhibits a net electroosmotic flow in the direction of the flow of $\mathrm{Cl}^{-}$. For the $1 \mathrm{M} \mathrm{MgCl}_{2}$ electrolyte, although the current of $\mathrm{Cl}^{-}$is greater than the current of $\mathrm{Mg}^{2+}$, the latter ion is much more effective dragging water molecules (its hydration layer is much more robust over time) so the net electroosmotic flow is in the direction of the flow of $\mathrm{Mg}^{2+}$ along the applied electric field.

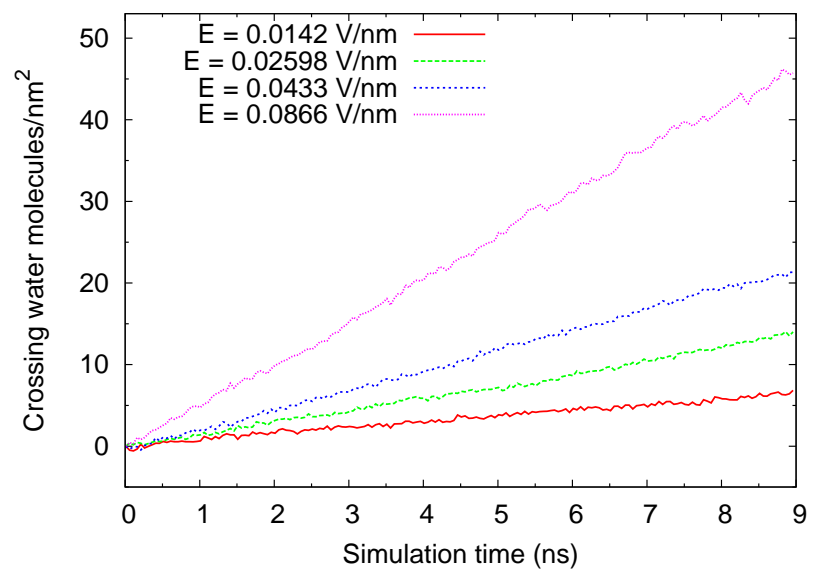

FIG. 6: Accumulated number of water molecules per unit area crossing a plane perpendicular to the applied electric field versus simulation time for the $1 \mathrm{M} \mathrm{MgCl}_{2}$ electrolyte. Different lines represent the flow of water for different values of the electric field. 

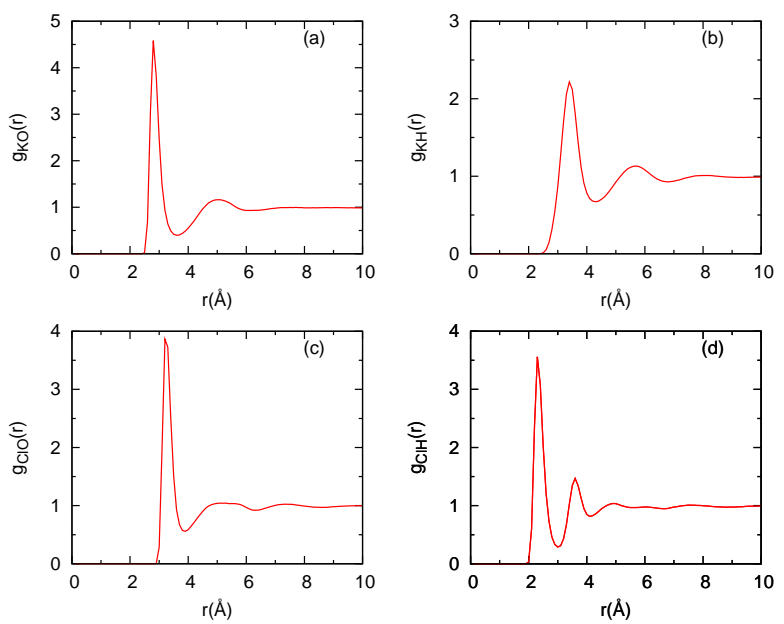

FIG. 7: The ion-oxygen and ion-hydrogen radial distribution functions for the $1 \mathrm{M} \mathrm{KCl}$ electrolyte
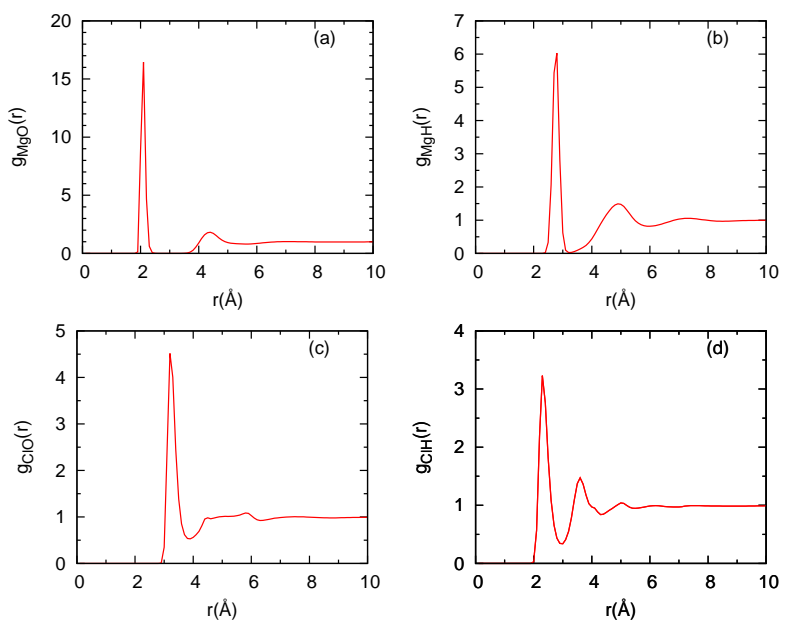

FIG. 8: The ion-oxygen and ion-hydrogen radial distribution functions for the $1 \mathrm{M} \mathrm{MgCl} 2$ electrolyte

\section{Concentration dependence of transport properties of $\mathrm{MgCl}_{2}$}

For the $\mathrm{MgCl}_{2}$ electrolyte we have also tested the dependency of the conduction properties of the electrolyte on its concentration, $c$. We have performed simulations at different concentrations of $\mathrm{MgCl}_{2}$ for a fixed value of the applied electric field $E=0.0433 \mathrm{~V} / \mathrm{nm}$ in a cubic simulation box of side $L \simeq 8 \mathrm{~nm}$ (see Table $\llbracket$ for precise values). The summary of the results are given in Table $\mathrm{VII}$ and Figures 9 and 10 . Here, for each value of the electrolyte concentration the conductivity has been obtained from a single point $(I, E)$, under the assumption that the electrolyte exhibits an ohmic behavior (suggested by the studies at $1 \mathrm{M}$ concentration, see Fig. 4). As expected, the electrical conductivity, $\kappa$, of the $\mathrm{MgCl}_{2}$ solution increases with increasing concentration, as can be seen
TABLE VII: Total current, electrical conductivity, ionic contributions to the total current, ratio of the anionic current over the cationic current and cationic transport numbers for different values of the concentration of the $\mathrm{MgCl}_{2}$ electrolyte.

\begin{tabular}{|c|cccccc|}
\hline $\mathrm{c}(\mathrm{M})$ & Itot $(\mathrm{nA})$ & $\kappa(\mathrm{S} / \mathrm{m})$ & $\mathrm{I}_{\mathrm{Mg}}(\mathrm{nA})$ & $\mathrm{I}_{\mathrm{Cl}}(\mathrm{nA})$ & $\mathrm{Ian} / \mathrm{Icat}$ & $\mathrm{t}_{+}$ \\
\hline 0.1 & 8.0 & 3.01 & 4.0 & 4.0 & 1.00 & 0.5 \\
0.3 & 19.97 & 7.55 & 9.42 & 10.54 & 1.12 & 0.47 \\
0.5 & 28.79 & 10.97 & 12.90 & 15.88 & 1.23 & 0.45 \\
1.0 & 31.70 & 12.25 & 13.66 & 18.05 & 1.32 & 0.43 \\
\hline
\end{tabular}

in Table VII and Fig. 9. In Fig. 9, the conductivity of the electrolyte obtained from molecular dynamics simulations for each concentration is compared with experimental results ${ }^{21}$ and the Kohlrausch's limiting law. The results obtained from MD simulations reproduce well the dependency of the electrolyte conductivity on the concentration, as compared to the experimental tendency. Besides, the absolute values of the experimental and simulated conductivities agree well with each other, especially for high and low salt conditions. In Fig. 9 we represent the ratio of the anionic current over the cationic current as a function of the $\mathrm{MgCl}_{2}$ concentration. The results from MD simulations indicate that the contribution of the anions becomes more prominent with increasing concentration of electrolyte. This tendency agrees with the experimental behaviour for $2: 1$ electrolytes $^{20}$.

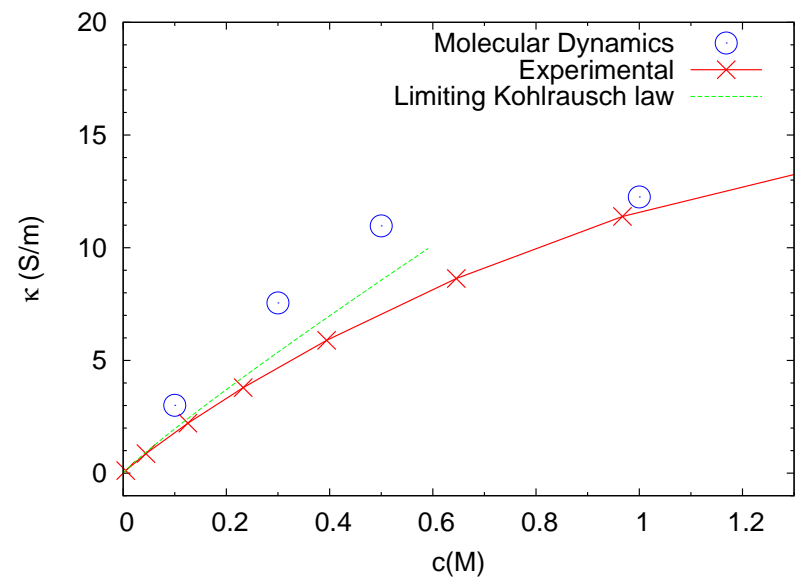

FIG. 9: Electric conductivity of a $\mathrm{MgCl}_{2}$ electrolyte versus its salt concentration for an electric field $E=43.3 \mathrm{mV} / \mathrm{nm}$. Blue dots represent results obtained from MD simulations, red crosses experimental data $^{21}$, and the green dashed line is Kohlrausch's limiting law ${ }^{20}$.

\section{Transport properties of mixture composed of $1 \mathrm{M} \mathrm{KCl}$ and $1 \mathrm{M} \mathrm{MgCl} 2$}

We have also studied the transport properties of an electrolyte composed of $1 \mathrm{M} \mathrm{KCl}$ and $1 \mathrm{M} \mathrm{MgCl}_{2}$. The addition of monovalent ions to a system immersed in a multivalent electrolyte is sometimes used experimentally to screen the electric charge of the multivalent ions ${ }^{-1}$. This effect is also used 


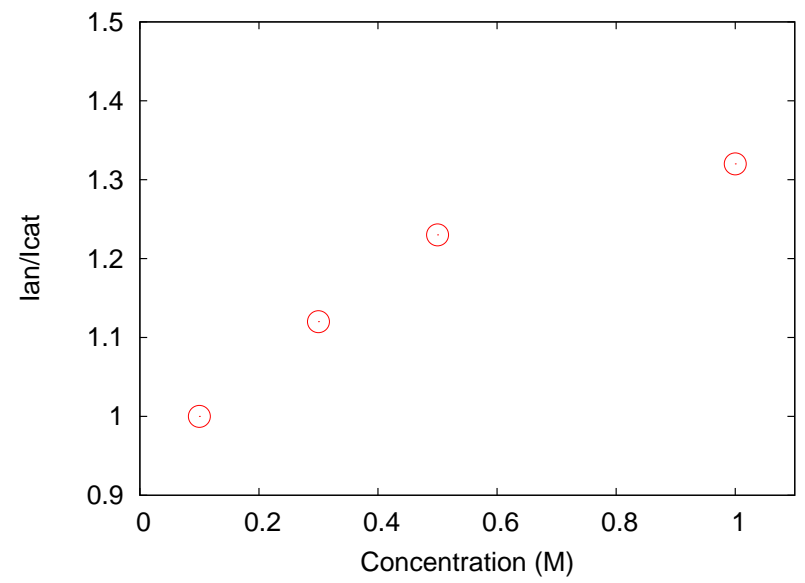

FIG. 10: Ratio of the contribution to the electric current of a $\mathrm{MgCl}_{2}$ electrolyte by anions and cations versus its bulk concentration when an electric field $E=43.3 \mathrm{mV} / \mathrm{nm}$ is applied.

in experiment and simulations to determine whether electrostatic correlations are responsible for a certain macroscopic effect ${ }^{11,32,33}$.

The analysis is similar to the analysis done for the previous electrolytes. The electric currents caused by the drift of ions have been obtained for four different values of the applied electric field. In this case, a single cubic box of size $L=7.72 \mathrm{~nm}$ was employed. The results for the conductivity are summarized in Fig. 11, which results in a value for the conductivity $\kappa=14.8 \mathrm{~S} / \mathrm{m}$. Our own experimental measurements give $\kappa=16.83 \pm 0.01 \mathrm{~S} / \mathrm{m}$. The different contribution to the total current of the different ions, as well as the cationic transport number are given in Table VIIIf for every value of the applied electric field.

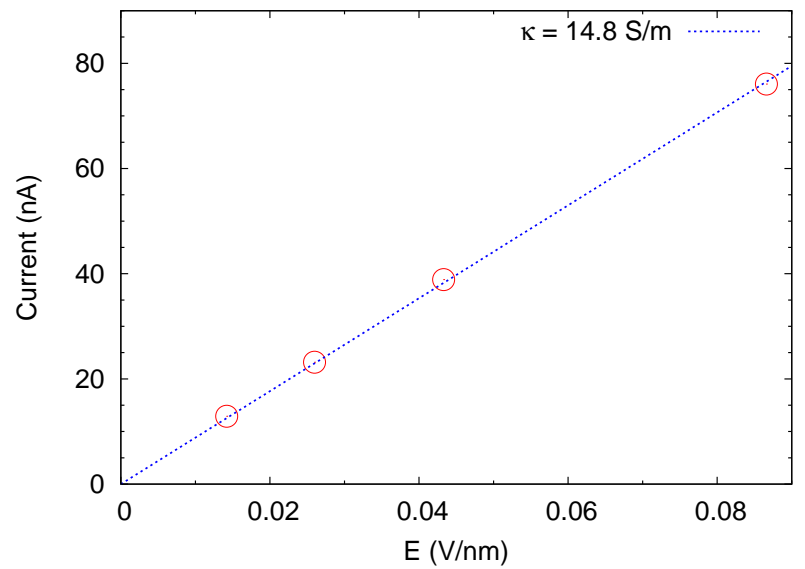

FIG. 11: MD results for the electric current versus applied electric field for an electrolyte composed of $1 \mathrm{M} \mathrm{KCl}$ and $1 \mathrm{M} \mathrm{MgCl} 2$ in a cubic simulation box of $L=7.72 \mathrm{~nm}$. The points correspond to simulation data computed from Eq. (1) and lines are fits of the data to Eq. (2) (fit gives $\kappa=14.8 \mathrm{~S} / \mathrm{m}$ )
TABLE VIII: Ionic contributions to the total current, ratio of the anionic current over the cationic current and cationic transport numbers for different values of the applied electric field in the case of an electrolyte composed by $1 \mathrm{M} \mathrm{MgCl} 2$ and $1 \mathrm{M} \mathrm{KCl}$. All these quantities are calculated in the frame of reference of the moving fluid.

\begin{tabular}{|c|c|c|c|c|c|}
\hline \hline Electric field $(\mathrm{V} / \mathrm{nm})$ & $\mathrm{I}_{\mathrm{Cl}}(\mathrm{nA})$ & $\mathrm{I}_{\mathrm{K}}(\mathrm{nA})$ & $\mathrm{I}_{\mathrm{Mg}}(\mathrm{nA})$ & $\mathrm{I}_{\mathrm{an}} / \mathrm{I}_{\mathrm{cat}}$ & $\mathrm{t}_{+}$ \\
\hline 14.2 & 7.6 & 3.27 & 2.04 & 1.43 & 0.41 \\
25.98 & 18.14 & 5.12 & 4.98 & 1.30 & 0.43 \\
43.3 & 21.69 & 9.29 & 7.89 & 1.26 & 0.44 \\
86.6 & 43.36 & 17.11 & 15.59 & 1.32 & 0.43 \\
\hline
\end{tabular}

\section{Electroosmotic flow}

The results for the net flow of water induced by the ionic current are given in Fig. 12, in which the accumulated number of water molecules per unit area crossing a plane perpendicular to the electric field is represented as a function of simulation time. The flux of water molecules for each electric field is given in Table III. Similarly to the case of the $1 \mathrm{M} \mathrm{MgCl}_{2}$ electrolyte, the net electroosmotic flow is in the direction of the flow of cations, in spite of being smaller than the net flow of anions (see Table VIII). We interpret this result along the same lines as with the case of $1 \mathrm{M} \mathrm{MgCl}_{2}$ electrolyte. As it is shown in Table VI, the hydration layer of $\mathrm{Mg}^{2+}$ is much more robust than the hydration of $\mathrm{Cl}^{-}$and $\mathrm{K}^{+}$, so the electroosmotic flow induced by the flow of $\mathrm{Mg}^{2+}$ dominates.

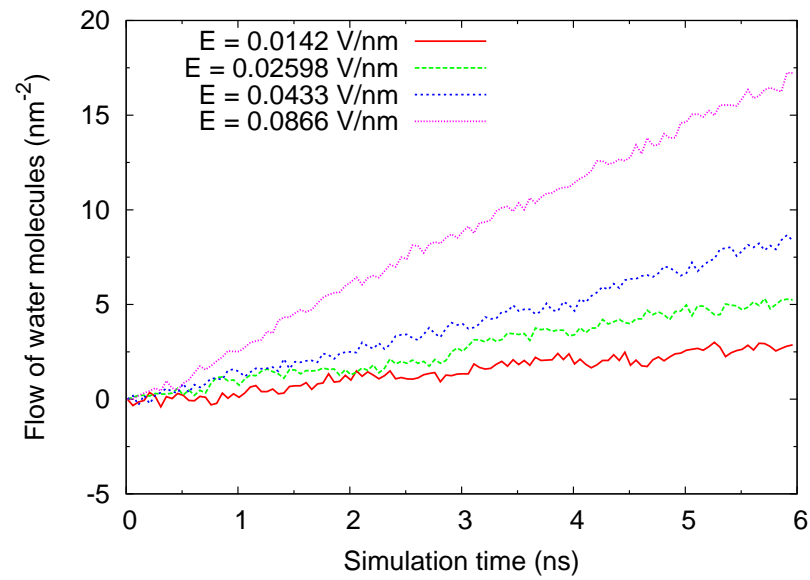

FIG. 12: Accumulated number of water molecules per unit area crossing a plane perpendicular to the applied electric field versus simulation time for an electrolyte composed of $1 \mathrm{M} \mathrm{MgCl}_{2}$ and $1 \mathrm{M} \mathrm{KCl}$. Different lines represent the flow of water for different values of the electric field.

\section{Transport properties of $1 \mathrm{M} \mathrm{LaCl}_{3}$}

The lanthanum cation $\mathrm{La}^{3+}$ is a highly charged and polarizable ion which, for most problems, requires the use of $\mathrm{Ab}$ 
TABLE IX: Ionic contributions to the total current, ratio of the anionic current over the cationic current and cationic transport numbers for different values of the applied electric field in the case of the $1 \mathrm{M}$ $\mathrm{LaCl}_{3}$ electrolyte, with $L=7.71 \mathrm{~nm}$. All these quantities are calculated in the frame of reference of the moving fluid.

\begin{tabular}{|c|c|c|c|c|}
\hline \hline Electric field $(\mathrm{V} / \mathrm{nm})$ & $\mathrm{I}_{\mathrm{Cl}}(\mathrm{nA})$ & $\mathrm{I}_{\mathrm{La}}(\mathrm{nA})$ & $\mathrm{I}_{\mathrm{Cl}} / \mathrm{I}_{\mathrm{La}}$ & $\mathrm{t}_{+}$ \\
\hline 14.2 & 5.51 & 5.00 & 1.10 & 0.48 \\
25.98 & 10.72 & 9.89 & 1.13 & 0.47 \\
43.3 & 17.55 & 14.94 & 1.17 & 0.46 \\
86.6 & 35.00 & 31.06 & 1.12 & 0.47 \\
\hline
\end{tabular}

initio calculations or polararizable force fields to properly describe its interaction with water and ions 34 . However, there are systems for which only its electric charge and size are relevant to describe certain electrokinetic phenomena ${ }^{33}$. For such cases, $\mathrm{La}^{3+}$ can be modeled as a charged Lennard-Jones particle and one can obtain the transport properties of a $\mathrm{LaCl}_{3}$ ionic solution by using classical molecular dynamics calculations. The Lennard-Jones parameters used here to describe the lanthanum cation $\mathrm{La}^{3+}$ were taken from Ref 35

The electrical transport properties of the $1 \mathrm{M} \mathrm{LaCl}_{3}$ electrolyte in the presence of an external electric field are obtained using molecular dynamics simulations which employ a nonpolarizable force field (described above). The summary of the results is given in Fig 13 and Table IX. The electrolyte shows an ohmic behaviour (see Fig 13, with an electrical conductivity of $\kappa=12.8 \mathrm{~S} / \mathrm{m}$. Considering the limitations of the description, this value is quite satisfactory compared to the experimental value of $\kappa_{\exp }=15.3 \mathrm{~S} / \mathrm{m}^{36}$. The ionic contribution, the ratio of currents and the cationic transport number are given in Table IX for each value of the external electric field.

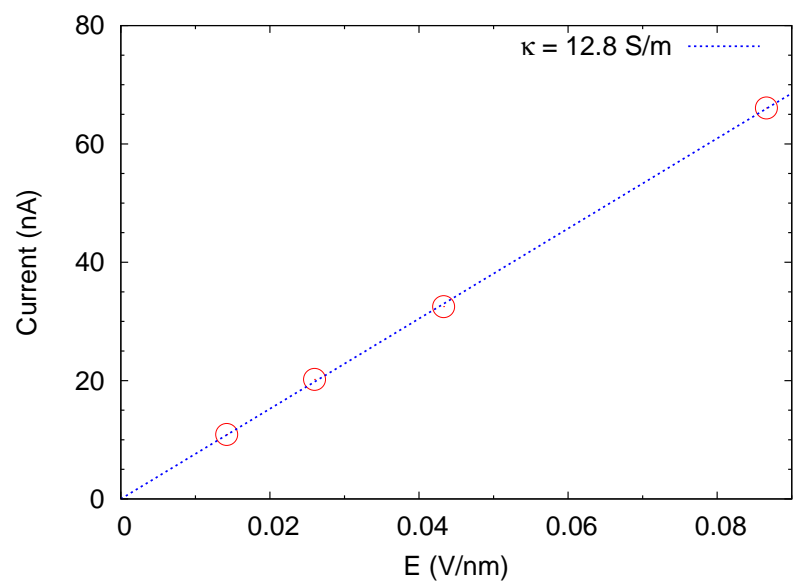

FIG. 13: MD results for the electric current versus applied electric field for a $1 \mathrm{M} \mathrm{LaCl}_{3}$ electrolyte in a cubic simulation box of $L=$ $7.71 \mathrm{~nm}$. The points correspond to simulation data computed from Eq.(1) and lines are fits of the data to Eq.(2) (fit gives $\kappa=12.8 \mathrm{~S} / \mathrm{m}$ )

\section{Electroosmotic flow}

The results for the net flow of water induced by the ionic current are given in Fig. 14, in which the accumulated number of water molecules per unit area crossing a plane perpendicular to the electric field is represented as a function of simulation time. The flux of water molecules for each electric field is given in Table II

The hydration properties of the $1 \mathrm{M} \mathrm{LaCl}_{3}$ electrolyte are summarized in Table VI In spite of the limitations of the non-polarizable model used to describe $\mathrm{La}^{3+}$, the values obtained for the hydration of $\mathrm{La}^{3+}$ are close to the values given in the literature (hydration $\sim 8-9$ and a residence time of hydrated water in the first coordination shell of $\sim 1 \mathrm{~ns}^{34}$ ). As showed in Fig. 14, the electroosmotic flow obtained for the $1 \mathrm{M} \mathrm{LaCl}{ }_{3}$ electrolyte is in the direction of the flow of chloride ions, opposite to the direction of the applied electric field. In this case, the higher hydration of $\mathrm{La}^{3+}$ is not enough to compensate the higher flow of chloride ions versus the flow of lanthanum cation $\mathrm{La}^{3+}$.

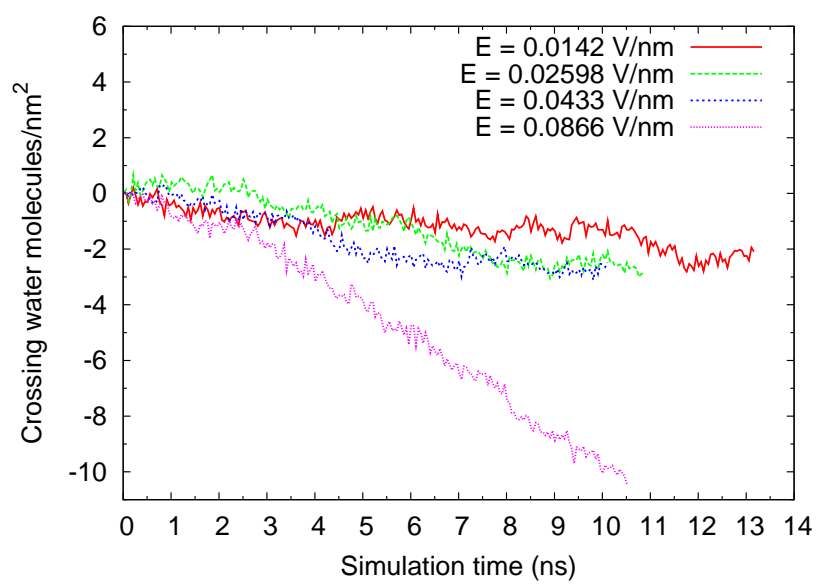

FIG. 14: Accumulated number of water molecules per unit area crossing a plane perpendicular to the applied electric field versus simulation time for an electrolyte composed of $1 \mathrm{M} \mathrm{LaCl}_{3}$. Different lines represent the flow of water for different values of the electric field.

\section{CONCLUSIONS}

Classical molecular dynamics simulations of various electrolytes $\left(\mathrm{KCl}, \mathrm{MgCl}_{2}, \mathrm{KCl}+\mathrm{MgCl}_{2}, \mathrm{LaCl}_{3}\right)$ in external electric fields were performed to study their transport properties. We have employed algorithms and parameters typically used in simulations of complex electrokinetic phenomena to test against experimental data their validity in the description of transport properties of electrolytes. The electrical conductivity and transport numbers (ionic contributions to the total current) of electrolytes containing monovalent $(1 \mathrm{M} \mathrm{KCl})$, divalent $(1 \mathrm{M} \mathrm{MgCl} 2,1 \mathrm{M} \mathrm{MgCl} 2+1 \mathrm{M} \mathrm{KCl})$, and trivalent $(1 \mathrm{M}$ $\mathrm{LaCl}_{3}$ ) cations were computed from the simulated ion trajectories. It is shown that in all cases the electrical conductivities 

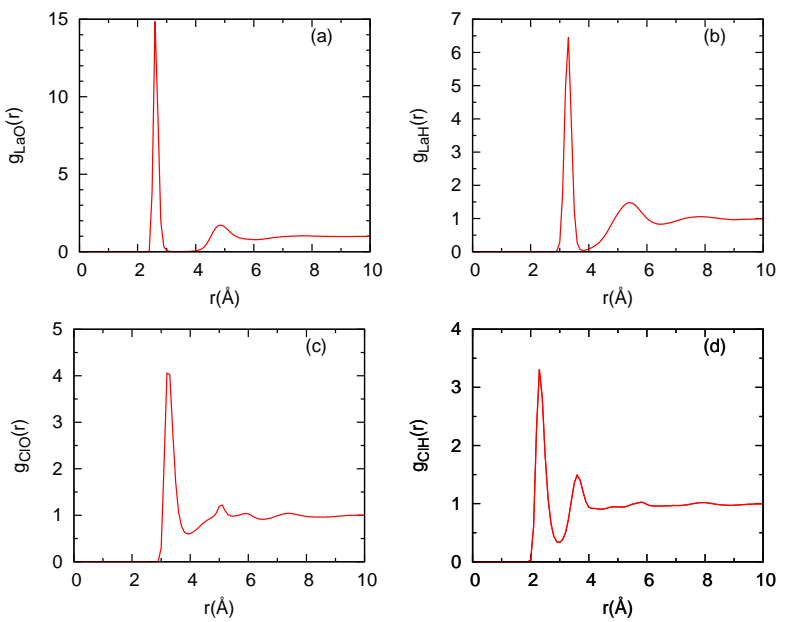

FIG. 15: The ion-oxygen and ion-hydrogen radial distribution functions for the $1 \mathrm{M} \mathrm{LaCl}_{3}$ electrolyte

obtained from the simulations are in good agreement with our own experimental measurements and values from the literature. Also, for the $\mathrm{MgCl}_{2}$ electrolyte, the dependence of the electrical conductivity on the electrolyte concentration was investigated and found that the MD results follow the experimental trend and give accurate results for the lowest $(0.1 \mathrm{M})$ and the highest concentrations (1M) studied. Transport numbers obtained for the $1 \mathrm{M} \mathrm{KCl}$ electrolyte agree well with the experimental values found in the literature. For the divalent electrolyte $1 \mathrm{M} \mathrm{MgCl}_{2}$, there is a significant discrepancy between the transport numbers obtained from MD simulations and the experimental values, probably due to the poor result for the self difusion coefficient for anions provided by the Lennard-Jones parameters used in the simulations. The effect of the simulation box size on the electrical transport properties was also explored. It is found that a big enough simulation box is needed to avoid spurious effects of the simulation.

The electroosmotic flow of water induced by the ionic flow has also been computed from the MD trajectories. It is shown that the direction and magnitude of the water flux depends on the electrolyte: while the flux is in the direction of the cation flow for electrolytes containing $\mathrm{Mg}^{2+}$, it is in the opposite direction and weaker for all the other cases. These results are interpreted with the help of the hydration properties of the ions, which are calculated and successfully compared with previous studies on the subject.

\section{ACKNOWLEDGMENTS}

This work is supported by the Spanish Government (grants FIS2009-13370-C02-02, FIS2007-60205 and CONSOLIDER-NANOSELECT-CSD2007-00041), Generalitat de Catalunya (2009SGR164) and Fundació Caixa Castelló-Bancaixa (P1-1A2009-13). C.C. is supported by the JAE doc program of the Spanish National Research Council (CSIC). The Supercomputing resources employed in this work were provided by the CESGA Supercomputing Center, Spain.
* Electronic address: ccalero@icmab.es

${ }^{1}$ F. H. J. van der Heyden, D. Stein, K. Besteman, S. G. Lemay and C. Dekker, Charge inversion at high ionic strength studied by streaming currents, Phys. Rev. Lett. 96 (2006),pp. 224502224505.

2 A. Alcaraz, E. M. Nestorovich, M. L. López, E. García, S.M. Bezrukov, V. Aguilella, Diffusion, Exclusion, and Specific Binding in a Large Channel: A Study of OmpF Selectivity Inversion, Biophysical Journal 96 (2009), 56-66.

${ }^{3}$ C. D. Lorenz, P. S. Crozier, J. A. Anderson and A. Travesset, Molecular dynamics of ionic transport and electrokinetic effects in realistic silica channels, J. Phys. Chem. C 112 (2008), 1022210232.

${ }^{4}$ B. Jönsson, A. Nonat, C. Labbez, B. Cabane, and H. Wennerström, Controlling the Cohesion of Cement Paste, Langmuir 21 (2005), 9211-9221.

5 A. Martín-Molina, J. A. Maroto-Centeno, R. Hidalgo-Álvarez and M. Quesada-Pérez, Charge reversal in real colloids: Experiments, theory and simulations, Colloids and Surfaces A 319 (2008), 103108.

${ }^{6}$ A. Aksimentiev and K. Schulten, Imaging $\alpha$-Hemolysin with Molecular Dynamics: Ionic Conductance, Osmotic Permeability, and the Electrostatic Potential Map, Biophys. J. 88 (2005), 37453761.

7 C. D. Lorenz and A. Travesset, Charge inversion of divalent ionic solutions in silica channels, Phys. Rev. E 75 (2007), 061202-
061206.

${ }^{8}$ B. Luan and Aleksei Aksimentiev, Electric and electrophoretic inversion of the DNA charge in multivalent electrolytes, Soft Matter 6 (2010), 243-246.

${ }^{9}$ E. R. Cruz-Chu, T. Ritz, Z. S. Siwy and K. Schulten, Molecular control of ionic conduction in polymer nanopores, Faraday Discussion, 143 (2009), 47-62.

${ }^{10}$ E. R. Cruz-Chu, A. Aksimentiev and K. Schulten, Water-silica force field for simulating nanodevices, J. Phys. Chem. B 110 (2006),21497-21508.

11 M. Aguilella-Arzo, C. Calero and J. Faraudo, Ionic correlations at the nanoscale: iversion of selectivity in a bio-nanochannel, arXiv: 1005.0278 v2 [cond-mat.mtrl-sci]

12 J. C. Phillips, R. Braun, W. Wang, J. Gumbart, E. Tajkhorshid, E. Villa, C. Chipot, R. D. Skeel, L. Kale, and K. Schulten, Scalable molecular dynamics with NAMD, J. Comp. Chem. 26 (2005), 1781-1802.

13 W.M. Gelbart, R.F. Bruinsma, P.A. Pincus and V.A. Parsegian, DNA-inspired electrostatics, Phys. Today 53 (2000), 38-44.

${ }^{14} \mathrm{~K}$. Besteman, K. van Eijk and S. Lemay, Charge inversion accompanies DNA condensation by multivalent ions, Nature Physics 3 (2007), 641-644.

$15 \mathrm{~J} . \mathrm{Yu}, \mathrm{T}$. Ha, and K. Schulten. Conformational model of the Holliday junction transition deduced from molecular dynamics simulations, Nuc. Acids Res. 32 (2004), 6683-6695.

16 B. Luan and A. Aksimentiev, DNA attraction in mono- and diva- 
lent electrolytes, J. Am. Chem. Soc. 130 (2008), 15754-15755.

17 D. Craig, M. Gao, K. Schulten, and V. Vogel, Structural insights into how the MIDAS ion stabilizes integrin binding to an RGD peptide under force, Structure 12 (2004), 2049-2058

18 F. C. J. M. van Veggel and D. N. Reinhoudt, New, Accurate Lennard-Jones Parameters for Trivalent Lanthanide Ions, Tested on [18]Crown-6, Chem. Eur. J. 5 (1999), 90-95.

${ }^{19}$ W. Humphrey, A. Dalke, and D. J. Schulten, VMD - Visual Molecular Dynamics, Molec. Graphics 14 (1996), 33-38.

${ }^{20}$ Robinson and Stokes, Electrolyte Solutions, Butterworths, London, 1959

21 S. Phang and R.H Stokes, Density, Viscosity, Conductance and Transference number of Concentrated Aqueous Magnesium Chloride at 25C, J. Sol. Chem. 9 (1980), 497-505.

22 D.G Miller, J.A Rard, L.B. Eppstein and J.G. Albright, $M u$ tual Diffusion Coefficients and Ionic transport Coefficients $I_{i j}$ of $\mathrm{MgCl}_{2}-\mathrm{H}_{2} \mathrm{O}$ at $25 \mathrm{C}$, J. Phys. Chem. 88 (1984), 5739-5748.

${ }^{23}$ Struis et al. An NMR Contribution to the Interpretation of the Dynamical Behavior of Water Molecules as a Function of the MgClp Concentration at 25 C, J. Phys. Chem. 93 (1989), 7943-7952.

24 D.G. Miller, Application of Irreversible Thermodynamics to Electrolyte Solutions. I Determination of Ionic Transport Coefficients $I_{i j}$ for isothermal Vector Transport Processes in Binary Electrolyte Systems, J. Phys. Chem 70 (1966), 2639-2659.

${ }^{25}$ S. Chowdhuri and A. Chandra, An NMR Contribution to the Interpretation of the Dynamical Behavior of Water Molecules as a Function of the MgClp Concentration at 25 C, J. Chem. Phys. 115 (2001), 3732-3741.

${ }^{26}$ K. M. Callahan, N. N. Casillas-Ituarte, M. Roeselová, H. C. Allen, and D. J. Tobias, Solvation of Magnesium Dication: Molecular Dynamics Simulation and Vibrational Spectroscopic Study of Magnesium Chloride in Aqueous Solutions, J. Phys. Chem. A 114 (2010), 5141-5148.

27 D. Jiao, C. King, A. Grossfield, T. A. Darden, P. Ren, Simulation of $\mathrm{Ca} 2+$ and $\mathrm{Mg} 2+$ Solvation Using Polarizable Atomic Multipole Potential J. Phys. Chem. B 110 (2006), 18553-18559.

${ }^{28}$ F. C. Lightstone, E. Schwegler, R. Q. Hood, F. Gygi, G. Galli, A first principles molecular dynamics simulations of the hydrated magnesium ion, Chem. Phys. Lett. 343 (2001), 549-555.

${ }^{29}$ K. Waizumi, I. Masuda, N. Fukushima, A molecular approach to the formation of $\mathrm{KCl}$ and $\mathrm{MgCl}+$ ion-pairs in aqueous solution by density functional calculations, Chem. Phys. Lett. 205 (1993), 317-323

${ }^{30}$ C. C. Pye, W. W. Rudolph, A molecular approach to the formation of $\mathrm{KCl}$ and $\mathrm{MgCl}+$ ion-pairs in aqueous solution by density functional calculations, J. Phys. Chem A 102 (1998), 9933-9943.

31 C. D. Cappa, J.D. Smith, B. M. Messer, R. C. Cohen, R. J. Saykally, Effects of Cations on the Hydrogen Bond Network of Liquid Water: New Results from X-ray Absorption Spectroscopy of Liquid Microjets, J. Phys. Chem. B 110 (2006), 5301-5309.

32 A. Martn-Molina, M. Quesada-Prez, F. Galisteo-Gonzlez and R. Hidalgo-lvarez, Probing charge inversion in model colloids: electrolyte mixtures of multi- and monovalent counterions, J. Phys.: Condens. Matter 15 (2003) S3475-S3483 (2003).

33 A. Martín-Molina, C. Rodríguez Beas, J. Faraudo, Charge Reversal in Anionic Liposomes: Experimental Demonstration and Molecular Origin, Phys. Rev. Lett. 104 (2010), 168103-168106.

${ }^{34}$ C. Clavaguéra, R. Pollet, J. M. Soudan, V. Brenner, and J. P. Dognon, Molecular Dynamics Study of the Hydration of Lanthanum(III) and Europium(III) Including Many-Body Effects, J. Phys. Chem B 109 (2005), 7614-7616.

35 F. C. J. M. van Veggel and D. N. Reinhoudt, New, Accurate Lennard-Jones Parameters for Trivalent Lanthanide Ions, Tested on [18]Crown-6, Chem. Eur. J. 5 (1999), 90-95.

${ }^{36}$ G. Jones and C. F. Bickford, The Conductance of Aqueous Solutions as a Function of the Concentration. I. Potassium Bromide and Lanthanum Chloride J. Am. Chem. Soc. 56 (1934), 602-611. 